Category: Teaching \& Learning at times of uncertainty

\title{
EASI framework: implementation of an online clinical and communication skills teaching
}

\author{
Nadarajah, V. ${ }^{1}$, Sow, C.F. ${ }^{1}$, Aznal S.S. ${ }^{1}$, Montagu, A. ${ }^{2}$, Boursicot, K. ${ }^{2}$, Er, H.M. ${ }^{3}$ \\ ${ }^{1}$ International Medical University, Malaysia, ${ }^{2}$ University of Adelaide, Australia, ${ }^{3}$ Duke-NUS Medical \\ School, Singapore
}

\begin{abstract}
A preparatory framework called EASI (Evaluate, Align, Student-centred, Implement and Improve) was developed with the aim of guiding the implementation for online Clinical and Communication Skills (CCS) learning. The workout example principle emphasises the importance of a structure framework in easing challenges, helps in implementation and monitoring for quality improvement. Although literature has some publications on converting face-to-face learning to online approaches, they do not focus or is limited to a specific aspect of CCS learning. The framework, when applied requires faculty to evaluate current resources, align sessions to learning outcomes with student-centred approaches and to continuously improve based on implementation experiences. Using the framework, we were able to generate various types of online CCS learning sessions for implementation in a short period of time due to the recent Covid-19 pandemic. Importantly we learnt a few lessons post-implementation from both students and faculty perspective that will be used for planning and delivery of future online CCS learning as healthcare systems and delivery are increasingly decentralised and widely distributed. Some of these lessons learned are managing student and faculty expectations, communicate the students' roles in online CCS learning and structured timetabling to avoid online fatigue.
\end{abstract}

Keywords: Clinical and communication skills, training framework 